\title{
Using Instagram Picture Features to Predict Users' Personality
}

\author{
Bruce Ferwerda ${ }^{(凶)}$, Markus Schedl, and Marko Tkalcic \\ Department of Computational Perception, \\ Johannes Kepler University, Altenberger Str. 69, 4040 Linz, Austria \\ \{bruce.ferwerda, markus.schedl, marko.tkalcic\}@jku.at \\ http://cp.jku.at
}

\begin{abstract}
Instagram is a popular social networking application, which allows photo-sharing and applying different photo filters to adjust the appearance of a picture. By applying these filters, users are able to create a style that they want to express to their audience. In this study we tried to infer personality traits from the way users manipulate the appearance of their pictures by applying filters to them. To investigate this relationship, we studied the relationship between picture features and personality traits. To collect data, we conducted an online survey where we asked participants to fill in a personality questionnaire, and grant us access to their Instagram account through the Instagram API. Among 113 participants and 22,398 extracted Instagram pictures, we found distinct picture features (e.g., relevant to hue, brightness, saturation) that are related to personality traits. Our findings suggest a relationship between personality traits and these picture features. Based on our findings, we also show that personality traits can be accurately predicted. This allow for new ways to extract personality traits from social media trails, and new ways to facilitate personalized systems.
\end{abstract}

Keywords: Instagram $\cdot$ Personality $\cdot$ Photo filters $\cdot$ Picture features

\section{Introduction}

Instagram is a popular mobile photo-sharing, and social networking application, with currently over 300 million active users a month, over 70 billion pictures shared, with an average of 70 million new pictures a day. ${ }^{1}$ Instagram is interconnected with an abundance of social networking sites (e.g., Facebook, Twitter, Tumblr, and Flickr) to let its users share their pictures on. In addition, it encourages users to apply filters to modify the color appearance of their pictures. At this moment Instagram offers 25 predefined photo filters that allow users to customize and modify their pictures to create the desired visual style.

The ease with which a photo filter can be applied allow users to express a personal style and create a seeming distinctiveness with the customized pictures.

\footnotetext{
1 https://instagram.com/press/ (accessed: 08/07/2015).

(C) Springer International Publishing Switzerland 2016

Q. Tian et al. (Eds.): MMM 2016, Part I, LNCS 9516, pp. 850-861, 2016.

DOI: $10.1007 / 978-3-319-27671-7-71$
} 
Through the shared content and the way of applying filters, users are able to reveal a lot about themselves to their social network. With that, the question arises: What do Instagram pictures tell about the user? Or more specifically: What do Instagram pictures say about the personality of the user?

Personality traits have shown to consist of cues to infer users' behavior, preference, and taste (e.g., $[9,26,28])$. Hence, knowing one's personality can provide important information for systems to create a personalized user experience. It can provide systems with estimations about user preferences, and avoid the use of extensive questionnaires or observations.

There is an increasing interest in implement personality in systems (e.g., $[8,11,29])$, and the implicit acquisition of personality from online behavior trails (e.g., Facebook [2,5,13,24,27], Twitter [12,25], Flickr [7], video blogs [3,4]). In this work we join the personality extraction research. We specifically focus on the relationship between the personality of Instagram users and the way they manipulate their pictures by using photo filters, in order to create a visual style.

Our work makes several contributions. We contribute to personality research, by showing relationships between personality traits and the visual style of users' Instagram pictures. Additionally, we contribute to new ways to extract personality from social media (i.e., Instagram). To the best of our knowledge, we are the first to investigate the relationship between personality traits and how users try to create a visual style by applying photo filters.

An online survey was conducted where we: (1) asked participants to fill in the widely used Big Five Inventory (BFI) personality questionnaire, and (2) grant us access to the content of their Instagram account. We extracted 22,398 Instagram pictures of 113 users, and analyzed them on several color-centric picture features (e.g., related to hue, saturation, value). We found distinct correlations between personality traits and picture features, and show that personality can be accurately predicted from the picture features. ${ }^{2}$

In the remainder of the paper we will continue with related work, materials, features, results, discussion, and conclusion.

\section{Related Work}

Personality has shown to be an enduring factor that can be related to a person's taste, preference, and interest (e.g., [9,26,28]). For example, Rawlings and Ciancarelli found relationships between personality traits and music genre preferences [26], while Tkalcic et al. found relationships between personality and classical music [28]. These relationships indicate that personality information can be used to create useful proxy measures for applications to cater to a more personalized service (e.g., [8,11,29]). For example, Tkalcic et al. propose to use personality to enhance the nearest-neighborhood measurement for overcoming the cold-start problem (i.e., recommending items to new users) in recommender systems [29]. Ferwerda et al. provide a way to use personality for adjusting the user interface of music applications to fit a user's music browsing style [11].

\footnotetext{
${ }^{2}$ Our preliminary results of this work can be found in [10].
} 
In order to measure personality, several models have been developed. The five-factor model (FFM) is the most well known and widely used one in the computing community [32], and categorizes personality into five general dimensions (traits), that describe personality in terms of: openness to experience, conscientiousness, extraversion, agreeableness, and neuroticism [21]. However, unless using extensive, and time-consuming questionnaires, acquiring personality traits is still a challenging task.

There is an emergent interest in how to implicitly acquire personality traits based on behavioral data (for an overview see [32]). Research has shown it is feasible to compute personality from behavioral data such as mobile phone usage (e.g., $[6,23])$, or with acoustic and visual cues through cameras and microphones (e.g., $[1,19,22]$ ). With the increasing connectedness of people, recent research has started to focus on personality acquisition from online behavior trails, such as, video blogs (vlogs) [3,4], Facebook behavior (e.g., [2,13,24,27]) and profile pictures [5], Twitter behavior (e.g., [12,25]), and Flickr pictures [7].

Little work has been done on personality extraction from pictures. Celli et al. focused on the content of Facebook profile pictures (e.g., facial close-ups, facial expressions, alone or with others) to extract personality [5]. Other work of Cristani et al. showed that personality can be extracted from the visual features of Flickr pictures [7]. Flickr attracts a lot of advanced photographers as an image hosting platform, and thereby consist of more serious and higher quality pictures. Instagram, on the other hand, targets snapshot pictures taken with the mobile phone, and puts emphasis on applying predefined filters. The different usage and interactions on Flickr and Instagram attract different audiences, and therefore personality prediction may be based on different cues. As Instagram is known for its photo filters to create certain effects, we decided to focus more on color-properties; how users manipulate their pictures with help of the filters to achieve a certain expression, rather then picture content.

\section{Materials}

To investigate the relationship between personality traits and picture features, we asked participants to fill in the 44-item BFI personality questionnaire (5point Likert scale; Disagree strongly - Agree strongly [16]). The questionnaire include questions that aggregate into the five basic personality traits of the FFM. The distribution of each personality trait can be found in Fig. 1. Additionally, we asked participants to grant us access to their Instagram account through the Instagram API, in order to crawl their pictures. From hereon, we define the picture-collection term as all the Instagram pictures of a single user.

We recruited 126 participants through Amazon Mechanical Turk, a popular recruitment tool for user-experiments [18]. Participation was restricted to those located in the United States, and also to those with a very good reputation ( $\geq 95 \%$ HIT approval rate and $\geq 1000$ HITs approved $)^{3}$ to avoid careless

\footnotetext{
${ }^{3}$ HITs (Human Intelligence Tasks) represent the assignments a user has participated in on Amazon Mechanical Turk prior to this study.
} 


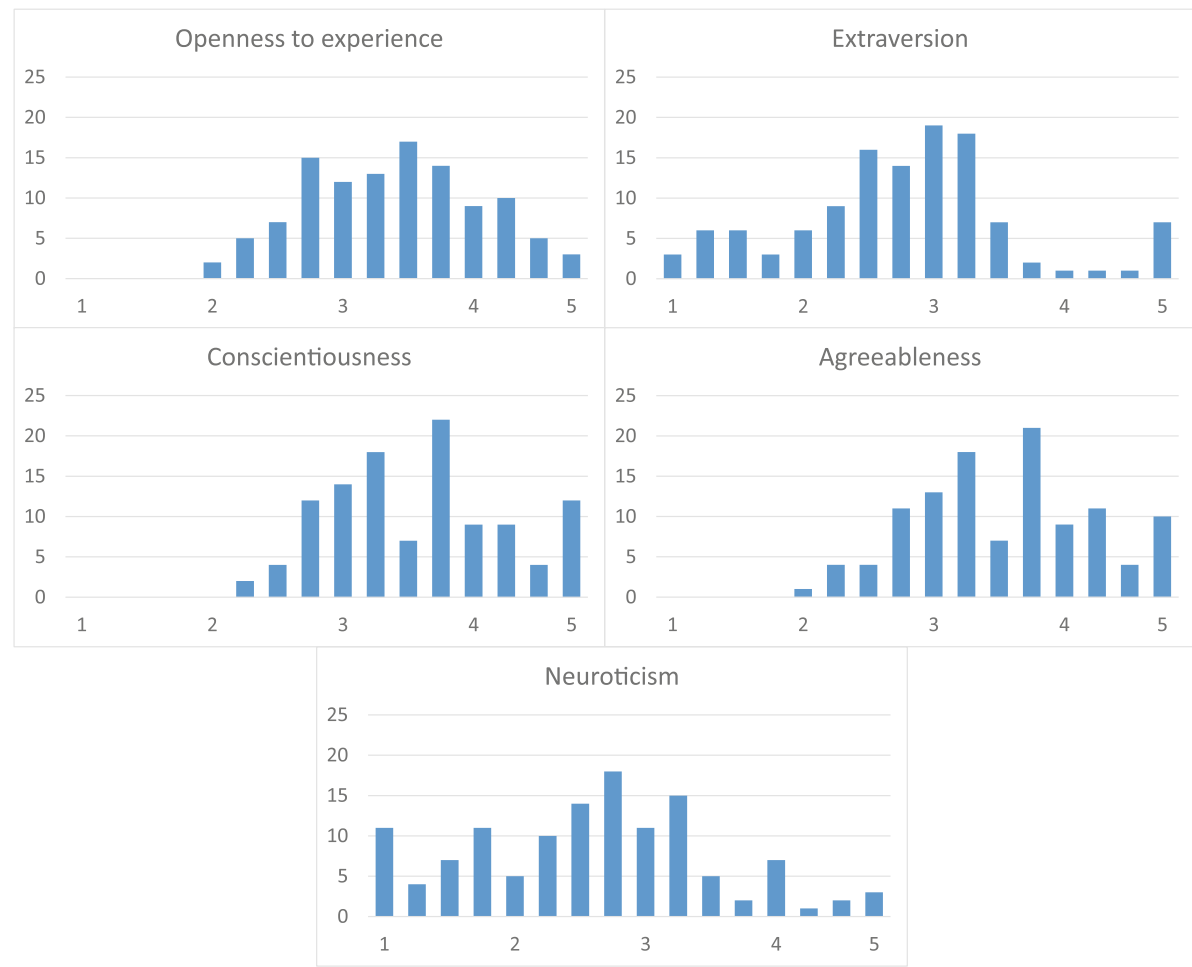

Fig. 1. Collected personality distribution for each personality trait.

contributions. Several comprehension-testing questions were used to filter out fake and careless entries. The Mahalanobis distance was calculated to check for outliers. This left us with 113 completed and valid responses. Age (18-64, median 30) and gender (54 male, 59 female) information indicated an adequate distribution. Pictures of each participant were crawled after the study. This resulted in a total of 22,398 pictures.

\section{Features}

As the goal in this study was to see how Instagram users manipulate their pictures with photo filters, we extracted mainly color related picture features. Based on the assumption that Instagram users' personality is manifested through the way photo filters are applied, a set of features that relate to color were selected based on the work of Machajdik and Hanbury [20]. For the color-centric features, the color space that is most closely related to the human visual system was selected. That is, the Hue-Saturation-Value (HSV) color space [30]. The $H$ parameter describes the hue (i.e., the color quality of each pixel) from orange through yellow, green, blue, violet to red, on a scale from 0 to 1 . The $S$ parameter 
describes how saturated the color is. That is, the share of white in a picture (high share of white means low saturation). The parameter $V$ (value) represents the brightness of the color. Beside the color-centric features, we additionally performed basic picture content analysis, by counting the number of faces and the number of people in each picture.

Hue-Related Features. We divided the range of the $H$ parameter into intervals that correspond to the hues: orange, yellow, green, blue, violet, and red. For each of these intervals we counted the number of pixels in an image that fall into this interval and divided it with the number of all pixels in the image. This yielded the share of the image surface that a hue covers.

Furthermore, we merged the cold colors (i.e., green, blue violet) and the warm colors (i.e., orange, red, yellow) into the respective shares across an image. On a user level, the features: orange, yellow, green, blue, violet, red, warm and cold, are the average values of the color shares among all the pictures of a user.

Saturation-Related Features. For each picture we calculated the average saturation and the variance. Images with low average saturation tend to be bleak and colorless, while pictures with high saturation have more vivid colors. Pictures with a high saturation variance tend to have both bleak and vivid colors. Here, we also divided the saturation axis into three equally spaced intervals and calculated the share of pixels that fall into each interval (low, mid, and high saturation). Pictures that have a high value in the low saturation tend to have more bleak colors, those with a high value in the mid saturation feature tend to have neither bleak nor vivid colors, and those that have a high value in the high saturation feature tend to have vivid colors across most of the image area.

Value-Related Features. For each image we calculated the average value (value mean) and variance (value variance) across all the pixels in the image. These features represent how light or dark a picture is and how much contrast it reveals, respectively. Pictures that have a high variance tend to have both dark and light areas, whereas pictures with a low variance tend to be equally bright across the image. Furthermore, we divided the value axis into three equally spaced intervals and counted the share of pixels that fall into each of these intervals (value low, mid, and high).

Pleasure-Arousal-Dominance (PAD). As the filters Instagram users can apply to their pictures, are intended to create certain expressions, we adopted the PAD model of Valdez and Merhabian [31], which contains general rules of the expression of pleasure, arousal, and dominance in a picture, and models these as a combination of brightness (value) and saturation levels:

1. Pleasure $=.69$ Value +.22 Saturation

2. Arousal $=-.31$ Value +.60 Saturation

3. Dominance $=-.76$ Value +.32 Saturation

Content-Based Features. In addition to the color-centric features, we computed two extra features for each user: (1) the average number of human faces 
across all the images of a user, and (2) the average number of full people bodies across all the images of a user.

To extract the number of faces in a picture, we used the Viola-Jones algorithm [33]. We trained the algorithm to recognize the number of faces in an image, by using the Haar-like features and the AdaBoost classifier. For extracting the number of full bodies we used the Histogram of Oriented Gradient (HOG) features with a Support Vector Machine (SVM) classifier. To achieve this, we employed Matlab's Computer Vision System Toolbox. ${ }^{4}$

\section{Results}

We divided the results section into two parts: the first part discusses the correlations we found, and the second part we discuss our personality regressor to predict personality traits based on the picture features.

\subsection{Correlations}

Although the main goal of this study was to investigate the relationship between personality and picture features, we decided to explore whether we could find correlations with personality and the usage of certain filters. We shortly discuss our results on the latter, and then continue with the correlations of the picture features.

Instagram Filters. Besides crawling the pictures, we also crawled descriptives about the filters each participant applied to their pictures, in order to explore whether certain personality traits are related to a more frequent use of some filters. Of the collected 22,398 pictures from 113 participants, 1487 unique filters were applied in total (this also include the "Normal" filter which means that no filter is applied). This brings that on average participants use 13 (13.15) different photo-filters to the pictures they upload to their picture-collection. Given that Instagram offers 25 different filters to apply, participants seem to use a whole range of filters. This is not so surprising, as the result of applying a filter depends on how the original picture looks like. So it is possible that the visual characteristics of a picture is eventually the same, but achieved by using different filters.

With using Pearson's correlation $(r \in[-1,1])$ to indicate the linear relationship between personality and photo filters, we found the following correlations: a positive correlation between the conscientiousness personality traits and the "Kelvin" filter $(r=.203, p=.044)$, a negative correlation between the agreeableness personality trait, the "Crema" $(r=-.205, p=.042)$ and the "Gotham" $(r=-.204, p=.042)$ filter. Additionally, we found a positive correlation between the neuroticism personality traits and the "Hudson" $(r=.224, p=.026)$ filter. These results imply that in general conscientiousness participants make more use

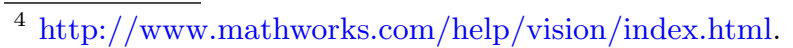


of the Kelvin filter, agreeable participants used less the Crema and Gotham filter, and neurotic participants applied more the Hudson filter. Besides these four significant correlations, there were no statistical significant correlations found with the remaining 21 photo filters. Given the low number of significant correlations that we found, and that the applied filter does not tell anything about how the end result looks like, we decided to not further pursue this direction, and continue our analyses focusing on the picture features.

Picture Features. For each picture from a crawled picture-collection (i.e., all the pictures of a participant's Instagram account), we extracted all the features that are described in Sect. 4 (Features). As the features in participants' picturecollection show a normal distribution, we calculated mean values for each feature to create a measurement of central tendency, to represent the whole picturecollection of each participant. The mean values of the features were used to calculate the correlation matrix (see Table 1). Pearson's correlation $(r \epsilon[-1,1])$ is reported to indicate the linear relationship between personality and picture features. The correlation matrix shows several features related with personality traits. ${ }^{5}$ We discuss the results related to each personality trait below.

Openness to Experience: Openness to experience was found to correlate with the saturation mean. This indicates that the pictures of open participants consist of more saturated, vivid colors. We also observed a positive correlation with the feature saturation variance, which means that open participants share pictures that have both vivid and bleak colors. Furthermore, a negative correlation with the feature value mean was found, indicating that open participants tend to share pictures that are low on brightness. This was further confirmed by the positive correlation on brightness low, and the negative correlation on value high. These correlations show that the pictures of open participants show more dark areas, and less bright areas. Also, a correlation was observed for the warm and cold features. Pictures of more open participants contained less warm colors (i.e., red, orange), but more cold colors (i.e., blue, green). Also, their pictures tend to express less pleasure, but more arousal and dominance. Additionally, their pictures consist in general of fewer faces and people.

Conscientiousness: A positive correlation was found between the saturation variance feature and conscientiousness. This indicates that conscientious participants more frequently shared pictures consisting of bleak and vivid colors.

Extraversion: We found correlations between the picture features and extraversion. Extraverts tend to create pictures with less red and orange, but with more green and blue tones. Additionally, their pictures tend to be darker (brightness low), but consist of both vivid and bleak colors (saturation variance). Also the emotion that the pictures of extraverts consist, are low on pleasure, but high on dominance.

\footnotetext{
${ }^{5}$ The magnitude of the correlations are commonly found in relationships between personality traits and social media trails (e.g., $[2,12,13,24,25,27])$.
} 
Table 1. Correlation Matrix of the picture features against the personality traits: (O)penness, (C)onscientiousness, (E)xtraversion, (A)greeableness, (N)euroticism.

\begin{tabular}{|c|c|c|c|c|c|}
\hline & $\mathrm{O}$ & $\mathrm{C}$ & $\mathrm{E}$ & $\mathrm{A}$ & $\mathrm{N}$ \\
\hline Red & -0.06 & 0.02 & $-0.17^{\wedge}$ & -0.05 & 0.03 \\
\hline Green & $0.17^{\wedge}$ & 0.14 & $0.23^{\wedge}$ & 0.03 & -0.12 \\
\hline Blue & -0.01 & 0 & $0.17^{\wedge}$ & 0.02 & -0.01 \\
\hline Yellow & 0.01 & 0.04 & 0.01 & 0.14 & -0.07 \\
\hline Orange & -0.03 & -0.07 & $-0.16^{\wedge}$ & -0.02 & 0.06 \\
\hline Violet & 0 & -0.06 & -0.09 & -0.07 & 0.06 \\
\hline Saturation mean & $0.16^{\wedge}$ & 0.06 & 0.03 & -0.04 & 0 \\
\hline Saturation variance & $0.20^{\wedge}$ & $0.16^{\wedge}$ & $0.19^{\wedge}$ & 0.10 & -0.05 \\
\hline Saturation low & -0.08 & -0.02 & 0.02 & 0.07 & 0.01 \\
\hline Saturation mid & 0.08 & -0.09 & 0.02 & 0.07 & 0.01 \\
\hline Saturation high & 0.13 & 0.10 & 0.04 & -0.01 & 0.01 \\
\hline Value mean & $-0.25^{*}$ & -0.10 & $-0.19^{\wedge}$ & -0.07 & $0.22^{\wedge}$ \\
\hline Value variance & 0.06 & 0 & 0 & -0.07 & 0.05 \\
\hline Value low & $0.28 * *$ & 0.09 & $0.16^{\wedge}$ & -0.05 & $-0.16^{\wedge}$ \\
\hline Value mid & -0.09 & 0.06 & 0.04 & $0.15^{\wedge}$ & -0.06 \\
\hline Value high & $-0.20^{\wedge}$ & -0.12 & $-0.18^{\wedge}$ & -0.08 & $0.21^{\wedge}$ \\
\hline Warm & $-0.05^{\wedge}$ & -0.04 & -0.20 & 0 & 0.03 \\
\hline Cold & $0.05^{\wedge}$ & 0.04 & 0.20 & 0 & -0.03 \\
\hline Pleasure & $-0.19^{\wedge}$ & -0.08 & $-0.18^{\wedge}$ & -0.09 & $0.22^{\wedge}$ \\
\hline Arousal & $0.23^{*}$ & 0.09 & 0.10 & 0 & -0.08 \\
\hline Dominance & $0.28 * *$ & 0.11 & $0.17^{\wedge}$ & 0.05 & $-0.18^{\wedge}$ \\
\hline \# of faces & $-0.16^{\wedge}$ & 0.03 & 0.11 & -0.11 & -0.03 \\
\hline \# of people & $-0.22^{\wedge}$ & -0.05 & -0.07 & -0.01 & 0.07 \\
\hline
\end{tabular}

Agreeableness: A positive correlation was found between agreeableness and the brightness mid feature. This means that the pictures of agreeable participants do not show emphasized bright or dark areas, but are more in between.

Neuroticism: A correlation was found on value mean, value low, and value high. The positive correlation with value mean indicate that participants scoring higher on the neurotic trait tend to share pictures that are high on brightness. This is also reflected in the value low (negative correlation) and value high (positive correlation) features. Additionally, correlations were found in the emotion expression of the pictures of extraverts. Result show that they adjust their pictures to express more pleasure but less dominance. 


\subsection{Personality Regressor}

Given the correlations that were found, we developed a personality regressor based on the features reported in Table 1. As prior work on prediction personality traits from Twitter behavior uses the same method of analyses [25], we compare our performance against their results. We trained our predictive model with several classifiers in Weka, with a 10 -fold cross-validation with 10 iterations. For each classifier we used, we report the root-mean-square error (RMSE) in Table 2, to indicate the root mean square difference between predicted and observed values. The RMSE of each personality trait relates to the $[1,5]$ score scale.

Table 2. Comparison of different classifiers to predict personality prediction compared to prior work of Quercia et al. [25]. Numbers in bold represent the results that outperform prior work. Root-mean-square error (RMSE) is reported $([1,5])$.

\begin{tabular}{l|l|l|l|l}
\hline & \multicolumn{4}{l}{ RMSE } \\
\hline Personality traits & $\begin{array}{l}\text { Radial basis } \\
\text { function network }\end{array}$ & $\begin{array}{l}\text { Random } \\
\text { forests }\end{array}$ & $\begin{array}{l}\text { M5 } \\
\text { rules }\end{array}$ & $\begin{array}{l}\text { M5 rules } \\
\text { Quercia et al. [25] }\end{array}$ \\
\hline Openness to experience & $\mathbf{0 . 6 8}$ & 0.71 & 0.77 & 0.69 \\
\hline Conscientiousness & $\mathbf{0 . 6 6}$ & $\mathbf{0 . 6 7}$ & $\mathbf{0 . 7 3}$ & 0.76 \\
\hline Extraversion & 0.90 & 0.95 & 0.96 & 0.88 \\
\hline Agreeableness & $\mathbf{0 . 6 9}$ & $\mathbf{0 . 7 1}$ & $\mathbf{0 . 7 8}$ & 0.79 \\
\hline Neuroticism & 0.95 & 1.01 & 0.97 & 0.85 \\
\hline
\end{tabular}

In line with prior work of Quercia et al. [25], we started to train our predictive model with the M5' rules [34]. Although our results do not outperform prior work on most facets, we do find similar trends: extraversion and neuroticism are the hardest personality traits to predict. As applying M5' rules did not result in any improvement, we applied the random forests classifier. Random forests are known to have a reasonable performance when the features consist of high amounts of noise [15]. The results show slight improvement over M5' rules in general, but for the neuroticism personality trait, the prediction got worse. Finally, we tried using the radial basis function (RBF) network, which is a neural network that has shown to work well on smaller datasets [17]. Results show that the RBF network outperforms the M5' rules, as well as the random forest classifier. Compared to prior work, the RBF network outperforms prediction of openness to experience, conscientiousness, and agreeableness. Also with the RBF network, predictions is most difficult for extraversion and neuroticism. Although we do not outperform prior work on extraversion and conscientiousness, our results do not differ much. In general, we show that with picture features we can achieve better personality prediction than prior work on Twitter data.

\section{Discussion}

We found Instagram picture features to be correlated with personality. A summary and interpretation of the picture features can be found in Table 3. We found 
Table 3. Interpretation and summary of the correlations found between personality traits and picture properties. The properties apply for the pictures of participants who score high in the respective personality trait.

\begin{tabular}{l|l}
\hline Personality & Picture properties \\
\hline Openness to experience & $\begin{array}{l}\text { More green tones, lower in brightness, higher in saturation, } \\
\text { more cold colors, fewer faces and people }\end{array}$ \\
\hline Conscientiousness & Mix of saturated and unsaturated colors \\
\hline Extraversion & $\begin{array}{l}\text { More green and blue tones, lower in brightness, mix of } \\
\text { saturated and unsaturated colors }\end{array}$ \\
\hline Agreeableness & Fewer dark and bright areas \\
\hline Neuroticism & Higher in brightness \\
\hline
\end{tabular}

that most correlations appear in the openness to experience personality trait. Even though, less and weaker correlations were found for the other personality traits, we were still able to observe distinct correlations.

Based on the identified correlations between personality and picture features, we created a personality regressor. The results that we achieved with our prediction model show that personality can be accurately predicted from picture features on Instagram. The results of the personality regressor show similar patterns as prior work on personality extraction from social media (i.e., Twitter) [25], and were able to outperform in predicting most of the personality traits. We found that the easiest and most successful prediction are for the openness to experience, conscientiousness, and agreeableness personality traits, whereas the more difficult traits are extraversion and neuroticism.

\section{Future Work and Limitations}

Our study contains limitations that need to be considered. Although we were able to obtain a fair amount of Instagram pictures $(n=22,398)$, our personality measurement was limited to 113 participants. Given that we only had personality information of 113 participants to find relationships with picture features, results would benefit from a bigger sample size.

In this study we solely focused on participants based in the United States. However, color interpretation and meaning could be influenced by cultural factors. Therefore, cultures could engage in different behavior of picture taking [14] and applying filters. Future work should address this. Furthermore, we decided to mainly focus on the color-centric features of the pictures we crawled, as Instagram's main focus is on applying photo filters. However, content analyses of the pictures would be an interesting next step to conduct.

\section{Conclusion}

With this study we show that personality of Instagram users can be accurately predicted from color-centric features of the pictures they post. Being able to 
implicitly extract personality from social media trails, gives possibilities to facilitate systems in order to provide a personalized experience. For example, it can help recommender systems to overcome the cold-start problem.

Acknowledgments. This research is supported by the Austrian Science Fund (FWF): P25655; and by the EU FP7/'13-'16 through the PHENICX project, grant agreement no. 601166 .

\section{References}

1. Aran, O., Gatica-Perez, D.: Cross-domain personality prediction: from video blogs to small group meetings. In: Proceedings of the 15th ACM on International Conference on Multimodal Interaction, pp. 127-130. ACM (2013)

2. Back, M.D., Stopfer, J.M., Vazire, S., Gaddis, S., Schmukle, S.C., Egloff, B., Gosling, S.D.: Facebook profiles reflect actual personality, not self-idealization. Psychol. Sci. 21, 372-374 (2010)

3. Biel, J.I., Aran, O., Gatica-Perez, D.: You are known by how you vlog: personality impressions and nonverbal behavior in youtube. In: ICWSM. Citeseer (2011)

4. Biel, J.I., Gatica-Perez, D.: The youtube lens: crowdsourced personality impressions and audiovisual analysis of vlogs. IEEE Trans. Multimedia 15(1), 41-55 (2013)

5. Celli, F., Bruni, E., Lepri, B.: Automatic personality and interaction style recognition from facebook profile pictures. In: Proceedings of the ACM International Conference on Multimedia, pp. 1101-1104. ACM (2014)

6. Chittaranjan, G., Blom, J., Gatica-Perez, D.: Mining large-scale smartphone data for personality studies. Pers. Ubiquit. Comput. 17(3), 433-450 (2013)

7. Cristani, M., Vinciarelli, A., Segalin, C., Perina, A.: Unveiling the multimedia unconscious: implicit cognitive processes and multimedia content analysis. In: Proceedings of the 21st ACM International Conference on Multimedia (2013)

8. Ferwerda, B., Schedl, M.: Enhancing music recommender systems with personality information and emotional states: a proposal. In: Proceedings of the EMPIRE Workshop (2014)

9. Ferwerda, B., Schedl, M., Tkalcic, M.: Personality \& emotional states: understanding users' music listening needs. In: UMAP 2015 Extended Proceedings (2015)

10. Ferwerda, B., Schedl, M., Tkalcic, M.: Predicting personality traits with instagram pictures. In: Proceedings of the 3rd Workshop on Emotions and Personality in Personalized Systems 2015, pp. 7-10. ACM (2015)

11. Ferwerda, B., Yang, E., Schedl, M., Tkalcic, M.: Personality traits predict music taxonomy preferences. In: CHI 2015 Extended Abstracts. ACM (2015)

12. Golbeck, J., Robles, C., Edmondson, M., Turner, K.: Predicting personality from twitter. In: IEEE Third Conference on Social Computing, pp. 149-156 (2011)

13. Gosling, S.D., Gaddis, S., Vazire, S., et al.: Personality impressions based on facebook profiles. In: ICWSM (2007)

14. Huang, C.M., Park, D.: Cultural influences on facebook photographs. Int. J. Psychol. 48(3), 334-343 (2013)

15. Humston, E.M., Knowles, J.D., McShea, A., Synovec, R.E.: Quantitative assessment of moisture damage for cacao bean quality using two-dimensional gas chromatography combined with time-of-flight mass spectrometry and chemometrics. J. Chromatogr. A 1217(12), 1963-1970 (2010) 
16. John, O.P., Donahue, E.M., Kentle, R.L.: The Big Five Inventory: Institute of Personality and Social Research. UC Berkeley, CA (1991)

17. Khot, L.R., Panigrahi, S., Doetkott, C., Chang, Y., Glower, J., Amamcharla, J., Logue, C., Sherwood, J.: Evaluation of technique to overcome small dataset problems during neural-network based contamination classification of packaged beef using integrated olfactory sensor system. LWT-Food Sci. Technol. 45(2), 233-240 (2012)

18. Kittur, A., Chi, E.H., Suh, B.: Crowdsourcing user studies with mechanical turk. In: Proceedings of the SIGCHI Conference on Human Factors in Computing Systems, pp. 453-456. ACM (2008)

19. Lepri, B., Subramanian, R., Kalimeri, K., Staiano, J., Pianesi, F., Sebe, N.: Connecting meeting behavior with extraversion - a systematic study. IEEE Trans. Affect. Comput. 3(4), 443-455 (2012)

20. Machajdik, J., Hanbury, A.: Affective image classification using features inspired by psychology and art theory. In: Proceedings of the International Conference on Multimedia, pp. 83-92. ACM (2010)

21. McCrae, R.R., John, O.P.: An introduction to the five-factor model and its applications. J. Pers. 60(2), 175-215 (1992)

22. Mohammadi, G., Vinciarelli, A.: Automatic personality perception: prediction of trait attribution based on prosodic features. IEEE Trans. Affect. Comput. 3(3), 273-284 (2012)

23. de Montjoye, Y.-A., Quoidbach, J., Robic, F., Pentland, A.S.: Predicting personality using novel mobile phone-based metrics. In: Greenberg, A.M., Kennedy, W.G., Bos, N.D. (eds.) SBP 2013. LNCS, vol. 7812, pp. 48-55. Springer, Heidelberg (2013)

24. Park, G., Schwartz, H.A., Eichstaedt, J.C., Kern, M.L., Kosinski, M., Stillwell, D.J., Ungar, L.H., Seligman, M.E.: Automatic personality assessment through social media language (2014)

25. Quercia, D., Kosinski, M., Stillwell, D., Crowcroft, J.: Our twitter profiles, our selves: predicting personality with twitter. In: IEEE Third Conference on Social Computing, pp. 180-185 (2011)

26. Rawlings, D., Ciancarelli, V.: Music preference and the five-factor model of the neo personality inventory. Psychol. Music 25(2), 120-132 (1997)

27. Ross, C., Orr, E.S., Sisic, M., Arseneault, J.M., Simmering, M.G., Orr, R.R.: Personality and motivations associated with facebook use. Comput. Hum. Behav. 25, $578-586(2009)$

28. Tkalčič, M., Ferwerda, B., Hauger, D., Schedl, M.: Personality correlates for digital concert program notes. In: Ricci, F., Bontcheva, K., Conlan, O., Lawless, S. (eds.) UMAP 2015. LNCS, vol. 9146, pp. 364-369. Springer, Heidelberg (2015)

29. Tkalcic, M., Kunaver, M., Košir, A., Tasic, J.: Addressing the new user problem with a personality based user similarity measure. In: DEMRA (2011)

30. Tkalcic, M., Tasic, J.: Colour spaces: perceptual, historical and applicational background. In: IEEE EUROCON, Computer as a Tool, pp. 304-308 (2003)

31. Valdez, P., Mehrabian, A.: Effects of color on emotions. J. Exp. Psychol. Gen. 123(4), 394 (1994)

32. Vinciarelli, A., Mohammadi, G.: A survey of personality computing. IEEE Trans. Affect. Comput. 5(3), 273-291 (2014)

33. Viola, P., Jones, M.J.: Robust real-time face detection. Int. J. Comput. Vis. 57(2), 137-154 (2004)

34. Witten, I.H., Frank, E.: Data Mining: Practical Machine Learning Tools and Techniques. Morgan Kaufmann, San Francisco (2005) 